\title{
An improved analytical method for triple stub matching (stubs in series)
}

\author{
Slobodan Babic ${ }^{1, *}$ and Cevdet Akyel ${ }^{2}$ \\ ${ }^{1}$ Independent Researcher, 53 Berlioz, 101, H3E 1N2, Montréal, Canada. \\ ${ }_{2}^{2}$ Département de Génie Électrique, École Polytechnique, C.P. 6079 Succ. Centre-Ville, QC H3C 3A7, Montréal, Canada.
}

Global Journal of Engineering and Technology Advances, 2021, 07(02), 103-123

Publication history: Received on 19 April 2021; revised on 22 May 2021; accepted on 24 May 20

Article DOI: https://doi.org/10.30574/gjeta.2021.7.2.0074

\begin{abstract}
In this paper we give the new improved analytical method of triple stub tuner for matching the load impedances to provide the maximum power transfer between a generator and a load. The stubs are connected in series with the line at the appropriate distances from the load. The characteristic impedances of the transmission line and the stabs are different. The paper points on the determination of the length for the first stub near the load. The limit lengths for the first and the second stub are found for which the matching is possible. The length of the third stub is directly obtained from the matching conditions. Event though there is not the unique solution for the triple stub matching, we shoved also the existence of the unique solutions under some conditions. The special cases are also treated. The results of this method are verified by using the standing wave ratio SWR. Event though there are not many papers on this subject this work could be useful for engineers and physicist which work in this domain.
\end{abstract}

Keywords: Impedance matching; Transmission line; Short or open stub; Triple stub in series

\section{Introduction}

In electronics, microwave and $R F$ engineering, the impedance matching is the practice of designing the input impedance of an electrical load or the output impedance of its corresponding signal source to maximize the power transfer or minimize signal reflection from the load.

In general, for the matching, the stubs are widely used to match any complex load to a transmission line. They consist of shorted or opened segments of the line, connected in parallel or in series with the line at the appropriate distances from the load and with their appropriate lengths. In the case of the single stub the distance from the load and its length are required for given the load impendence as well as the characteristic impedances of the line and the stub. Single stubs can match any load impendence to a transmission line, but the problem is a variable distance between the stab and the load. To overcome the drawbacks of the single-stub matching technique, the double-stub matching technique is employed. This is way the double stabs are preferable because they are inserted at predetermined locations. Thus, for given distance between stubs and the given position from the first stub and the load, the stub lengths are required. A disadvantage of double stubs is that they can't match all loads. To overcome this problem, triple stubs are used, [1]-[10]. With them all loads can be matched. The distances between the second and the third stub, the second and the first stub are given as well as the distance between the first stub and the load. The stub lengths are required. In this paper we treat the stubs in series. Even though there is no unique solution for triple stub matching we think that the presented method could be a useful contribution on this subject. Also, we found the unique solution in the triple stub matching under some conditions. As it was mentioned we will work with the different impedances everywhere. Still, all combinations either for short stubs or for open stubs can be treated with this approach. We propose this paper as the

\footnotetext{
${ }^{*}$ Corresponding author: Slobodan Babic

Independent Researcher, 53 Berlioz, 101, H3E 1N2, Montréal, Canada.

Copyright $(2021$ Author(s) retain the copyright of this article. This article is published under the terms of the Creative Commons Attribution Liscense 4.0.
} 
educational tool for the students and people which work in this domain because they can make the own MATLAB or MATHEMATICA codes using the formulas given in this work.

\section{Analytical method}

The load impedance $Z_{L}=A+j B,(A, B \in R)$ of the transmission line with the characteristic impendence $Z_{0}$ and without losses, is to be matched by a triple-stub tuner connected in parallel (Fig. 1). The first stub is distanced from the load by $d_{1}$. The distance between the first and the second stub is $d_{2}$ and the distance between the second and the third stub is $d_{3}$. Their characteristic impendences are respectively $Z_{S 1}=k_{1} Z_{0}, Z_{S 2}=k_{2} Z_{0}$ and $Z_{S 3}=k_{3} Z_{0}$. The lengths $l_{1}$, $l_{2}$ and $l_{3}$ are required to find. In this paper all impedances are normalized.

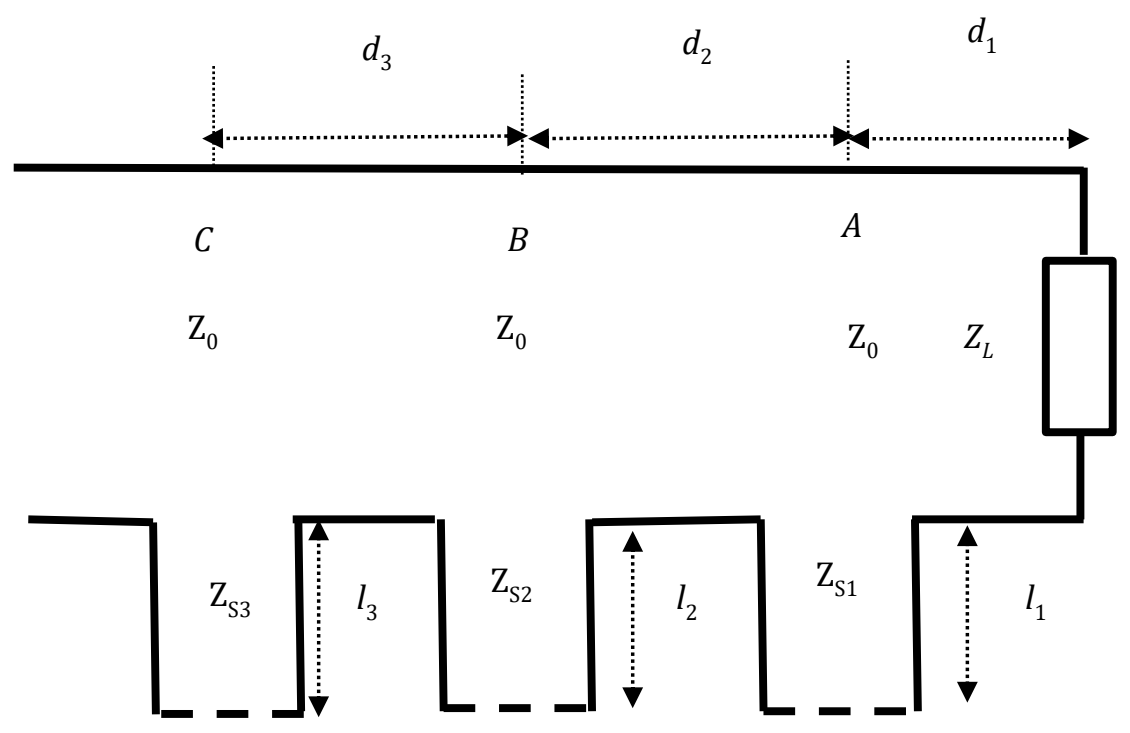

Figure 1 Triple short stubs in series (S/S/S; S/S/O; O/S/S; O/S/O/; S/O/S; S/O/O; O/O/S; O/O/O)

The normalized load admittance is,

$$
z_{L N}=p_{L}+j q_{L}
$$

where

$$
p_{L}=\frac{A}{Z_{0}}, q_{L}=-\frac{B}{Z_{0}}
$$

Let us calculate the normalized impedance at points $A$,

where

$$
z_{A}=z_{\left(d_{1}\right)}=\frac{z_{L N}+j \tan \left(\beta d_{1}\right)}{1+j \tan \left(\beta d_{1}\right) y_{L N}}=\frac{z_{L N}+j a}{1+j a z_{L N}}=\frac{z_{L N}+j a}{1+j a z_{L N}}=r_{L A}+j x_{L A}
$$

$$
\begin{gathered}
a=\tan \left(\beta d_{1}\right), a \in R \\
r_{L A}=\frac{p_{L}\left(1+a^{2}\right)}{\left(1-a q_{L}\right)^{2}+a^{2} p_{L}^{2}} \\
x_{L A}=\frac{a\left(1-p_{L}^{2}-q_{L}^{2}\right)+q_{L}\left(1-a^{2}\right)}{\left(1-a q_{L}\right)^{2}+a^{2} p_{L}^{2}}
\end{gathered}
$$


The total impedance at points $A$ is,

$$
z_{\text {TotalA }}=z_{A}+j z_{S 1}=r_{L A}+j\left(x_{L A}+x_{S 1}\right)=g_{L A}+j X_{1}
$$

where

$$
z_{S 1}=j x_{S 1}
$$

$$
X_{1}=x_{L A}+x_{S 1}
$$

$$
x_{S 1}=k_{1} b, x_{S 1}=k_{1} \tan \left(\beta l_{1}\right)=k_{1} b,
$$

$$
x_{S 1}=-\frac{k_{1}}{b}, \quad x_{S 1}=-k_{1} b \tan \left(\beta l_{1}\right)=-\frac{k_{1}}{b}
$$

$$
b=\tan \left(\beta l_{1}\right)
$$

The next step is the determination of the impedance at point $B$. Now, the impedance at the point $B$ is

$$
z_{B}=\frac{z_{\text {TotalA }}+j \tan \left(\beta d_{2}\right)}{1+j z_{\text {TotalA }} \tan \left(\beta d_{2}\right)}=\frac{z_{\text {TotalA }}+j m}{1+j z_{\text {TotalA }} m}=r_{L B}+j x_{L B}
$$

where

$$
m=\tan \left(\beta d_{2}\right)
$$

$$
\begin{aligned}
& r_{L B}=\frac{r_{L A}\left(1+m^{2}\right)}{\left(1-m X_{1}\right)^{2}+m^{2} r_{L A}^{2}} \\
& x_{L B}=\frac{m\left(1-r_{L A}^{2}-X_{1}^{2}\right)+X_{1}\left(1-m^{2}\right)}{\left(1-m X_{1}\right)^{2}+m^{2} r_{L A}^{2}}
\end{aligned}
$$

The total impedance at the point $B$ is,

$$
z_{\text {TotalB }}=z_{B}+j z_{S 2}=r_{L B}+j\left(x_{L B}+x_{S 2}\right)=r_{L B}+j X_{2}
$$

with

$$
X_{2}=x_{L B}+x_{S 2}
$$

where

$$
\begin{array}{ll}
x_{S 2}=k_{2} n, z_{S 2}=k_{2} \tan \left(\beta l_{2}\right)=k_{2} n, & \text { short circuit }\left(\frac{S}{C}\right) \\
x_{S 2}=-\frac{k_{2}}{n}, x=-k_{2} \operatorname{ctan}\left(\beta l_{2}\right)=-\frac{k_{2}}{n}, & \text { open circuit }\left(\frac{O}{C}\right)
\end{array}
$$

with $n=\tan \left(\beta l_{2}\right)$

The impedance at the point $C$ is,

$$
z_{C}=\frac{z_{\text {TotalB }}+j \tan \left(\beta d_{3}\right)}{1+j z_{\text {TotalB }} \tan \left(\beta d_{3}\right)}=\frac{z_{\text {TotalB }}+j p}{1+j z_{\text {TotalB }} p}=r_{L C}+j x_{L C}
$$


with

$$
p=\tan \left(\beta d_{3}\right)
$$

$$
\begin{aligned}
& r_{L C}=\frac{r_{L B}\left(1+p^{2}\right)}{\left(1-p X_{2}\right)^{2}+p^{2} r_{L B}^{2}} \\
& x_{L C}=\frac{p\left(1-r_{L}^{2}-X_{2}^{2}\right)+X_{2}\left(1-p^{2}\right)}{\left(1-p B_{2}\right)^{2}+p^{2} g_{L B}^{2}}
\end{aligned}
$$

The total admittance at the point $C$ is,

$$
z_{\text {TotalC }}=z+j z_{S 3}=r+j\left(x_{L C}+x_{S 3}\right)=r_{L C}+j X_{3}
$$

with

$$
X_{3}=x_{L C}+x_{S 3}
$$

The condition of the matching at the point $\boldsymbol{C}$ is,

$$
z_{\text {TotalC }}=1
$$

which gives,

$r_{L C}=1$ or

$$
\frac{r_{L B}\left(1+p^{2}\right)}{\left(1-p X_{2}\right)^{2}+p^{2} r_{L B}^{2}}=1
$$

and

$$
\begin{aligned}
& x_{L C}+x_{S 3}=0 \text {, or } \\
& x_{S 3}=-\frac{p\left(1-r_{L B}^{2}-X_{2}^{2}\right)+X\left(1-p^{2}\right)}{\left(1-p X_{2}\right)^{2}+p^{2} r_{L B}^{2}}
\end{aligned}
$$

where,

$$
\begin{array}{ll}
x_{S 3}=k_{3} q, x_{S 3}=k_{3} \tan \left(\beta l_{3}\right)=k_{3} q, & \left(\frac{S}{C}\right) \\
x_{S 3}=-\frac{k_{3}}{q}, z_{S 3}=-k_{3} \operatorname{ctan}\left(\beta l_{3}\right)=\frac{k_{3}}{q}, & \left(\frac{O}{C}\right)
\end{array}
$$

The next step is determination of all stub lengths.

From (19) we obtain,

$$
\left(X_{2}-\frac{1}{p}\right)^{2}+r_{L B}^{2}=r_{L B}^{2} \frac{r_{(p)}}{r_{L B}}
$$

whose solutions for $X_{2}$ are, 


$$
X_{2}^{(1,2)}=\frac{1}{p} \pm r_{L B} \sqrt{\frac{r_{(p)}}{r_{L B}}-1}
$$

where

$$
r_{(p)}=\frac{1+p^{2}}{p^{2}}
$$

Equation (24) can have the real solutions if,

$$
\frac{r_{\mathrm{r}(p)}}{r_{L B}}-1 \geq 0
$$

or,

$$
r_{L B} \leq r_{(p)}=\frac{1+p^{2}}{p^{2}}
$$

Also, equation (23) can be written in the form,

$$
r_{L B}^{2}-r_{L B} r_{(p)}+\left(X_{2}-\frac{1}{p}\right)^{2}=0
$$

whose solutions are,

$$
r_{L B(1,2)}=\frac{r_{(p)} \pm \sqrt{r_{(p)}^{2}-4\left(X_{2}-\frac{1}{p}\right)^{2}}}{2}
$$

The following condition must be satisfied,

$$
r_{(p)}^{2}-4\left(X_{2}-\frac{1}{p}\right)^{2} \geq 0
$$

which gives,

$$
-\frac{(p-1)^{2}}{2 p^{2}} \leq X_{2} \leq \frac{(p+1)^{2}}{2 p^{2}}
$$

or

$$
-r_{L B(i)}-\frac{(p-1)^{2}}{2 p^{2}} \leq X_{S 2}^{(i)} \leq-r_{L B(i)}+\frac{(p+1)^{2}}{2 p^{2}}
$$

This condition is important because it gives the limits for $X_{2}$ or the reactance for the second stub. Combining (7) and (26) we obtain,

$$
r_{L B}=\frac{r_{L A}\left(1+m^{2}\right)}{\left(1-m X_{1}\right)^{2}+m^{2} r_{L A}^{2}} \leq r_{(p)}
$$

or 


$$
\frac{r_{L A} r_{(m)}}{\left(X_{1}-\frac{1}{m}\right)^{2}+r_{L A}^{2}} \leq r_{(p)}
$$

where

$$
r_{(m)}=\frac{1+m^{2}}{m^{2}}
$$

Let's introduce the constant $Q$ as,

$$
Q=\frac{r_{(m)}}{r_{L A} r_{(p)}}=\frac{\left(1+m^{2}\right) p^{2}}{m^{2}\left(1+p^{2}\right) r_{L A}}>0, \forall m, p, r_{L A}
$$

The expression (30) can be written in the following form,

$$
\frac{r_{L A}^{2} Q}{\left(X_{1}-\frac{1}{m}\right)^{2}+r_{L A}^{2}} \leq 1
$$

This inequality presents the condition for finding the length of the first stub. Introducing the parameter $t \geq 1$, (33) can be written as,

$$
\frac{r_{L A}^{2} Q}{\left(X_{1}-\frac{1}{m}\right)^{2}+r_{L A}^{2}}=\frac{1}{t} \leq 1
$$

Let us take the limit of (34),

$$
\left(X_{1}-\frac{1}{m}\right)^{2}+r_{L A}^{2}=r_{L A}^{2} Q t
$$

or

$$
\left(X_{1}-\frac{1}{m}\right)^{2}=r_{L A}^{2}(Q t-1)
$$

I) $0<Q<1, t \geq 1$

I.1) If $1 \leq t \leq 1 / Q=r_{L A} \frac{r_{(p)}}{r_{(m)}}=t_{\max }$

$D=Q t-1=0$

that gives,

$$
\left(X_{1}-\frac{1}{m}\right)^{2}=0
$$

$$
X_{1}=\frac{1}{m}
$$

Equation (37) gives the reactance for the first stub,

$$
x_{S 1}=-x_{L A}+\frac{1}{m}
$$


One can see that $x_{S 1}$ does not depend on ' $t$ '. Moreover, the reactance for the second and the third stubs do not depend on ' $t$ ' by their definition. It is important because the unique triple stub matching is achieved for

$$
1 \leq t \leq t_{\max } \text { and } 0<Q<1
$$

Thus, there is the unique solution (37) or (38) for the triple stub matching with (36).

I.2) If $0<Q<1, t>1 / Q$

$$
D=Q t-1>0
$$

that gives

$$
X_{1}=\frac{1}{m}+r_{L A} \sqrt{Q t-1} \text { and } X_{1}=\frac{1}{m}-r_{L A} \sqrt{Q t-1}
$$

or

$$
x_{S 1}^{(1,2)}=-x_{L A}+\frac{1}{m} \pm r_{L A} \sqrt{Q t-1}
$$

with the condition,

$$
r_{L A} \leq \frac{\left(1+m^{2}\right) p^{2}}{m^{2}\left(1+p^{2}\right)} t
$$

II) $Q \geq 1, \quad t \geq 1$

For these values, this condition $Q t-1 \geq 0$ is satisfied automatically and the reactances $X_{1}^{(1,2)}$ or $x_{S 1}^{(1,2)}$ for the first stub are determined by (39) and (40) with condition (41) for $r_{L A}$,

Expressions (39) give the limits where the matching is possible for the first stub.

$$
X_{1} \geq \frac{1}{m}+r_{L A} \sqrt{Q t-1} \text { and } X_{1} \leq \frac{1}{m}-r \sqrt{Q t-1}
$$

From the previous analysis one can conclude that there is not the unique solution for

$$
\text { A) If } 0<Q<1, t>1 / Q \text { and }
$$

$$
\text { B) If } Q \geq 1, t \geq 1
$$

It could be an optimization problem. This is problem of the three variables where the lengths of the three stubs could be chosen to optimize the bandwidth of the matching.

The corresponding lengths for the first stub can be obtained for:

a) Short circuit (stub)

$$
x_{S 1}^{(1,2,3,4)}=k_{1} b_{\left(\frac{S}{C}\right) S 1}^{(1,2,3,4)}, \quad x_{S 1}^{(1)}=x_{S 1}^{(2)}, x_{S 1}^{(3)}=x_{S 1}^{(4)}
$$

or

$$
b_{\left(\frac{S}{C}\right) S 1}^{(1,2,3,4)}=\frac{x_{S 1}^{(1,2,3,4)}}{k_{1}}
$$




$$
l_{\left(\frac{S}{C}\right) S 1}^{(1,2,3,4)}=\frac{\lambda}{2 \pi} \operatorname{atan}\left[b_{\left(\frac{S}{C}\right)}^{(1,2,31,4)}\right]
$$

b) Open circuit (stub)

$$
x_{S 1}^{(1,2,3,4)}=-\frac{k_{1}}{b_{\left(\frac{o}{C}\right) S 1}^{(1,2,3)}}, \quad x_{S 1}^{(1)}=x_{S 1}^{(2)}, \quad x_{S 1}^{(3)}=x_{S 1}^{(4)}
$$

or

$$
-\frac{k_{1}}{x_{S 1}^{(1,2,3,4)}}=b_{\left(\frac{O}{C}\right) s 1}^{(1,2,3,4)}
$$

$$
l_{\left(\frac{O}{C}\right) s 1}^{(1,2,3,4)}=\frac{\lambda}{2 \pi} \operatorname{atan}\left[b_{\left(\frac{o}{C}\right) s 1}^{(1,2,3,4)}\right]
$$

From (41) it can be seen that

$$
X_{1}^{(1)}+X_{1}^{(2)}=\frac{2}{m}
$$

and

$$
\begin{aligned}
& r_{L B 1}=r_{L B 2}=\frac{r_{L A}\left(1+m^{2}\right)}{\left(1-m X_{1}^{(1)}\right)^{2}+m^{2} r_{L A}^{2}} \\
& x_{L B 1}=\frac{m\left(1-r_{L A}^{2}-X_{1}^{(1) 2}\right)+X_{1}^{(1)}\left(1-m^{2}\right)}{\left(1-m B_{1}^{(1)}\right)^{2}+m^{2} r_{L A}^{2}} \\
& x_{L B 2}=\frac{m\left(1-r_{L A}^{2}-X_{1}^{(2) 2}\right)+X_{1}^{(2)}\left(1-m^{2}\right)}{\left(1-m X_{1}^{(1)}\right)^{2}+m^{2} r_{L A}^{2}}
\end{aligned}
$$

Now, we simply find from (10) and (23) the reactance for the second stub,

$$
x_{S 2}^{(1,2,3,4)}=-x_{L B}^{(1,2)}+\frac{1}{p} \pm r_{L B}^{(1,2)} \sqrt{\frac{r_{(p)}}{r_{L B}^{(1,2)}}-1}
$$

where $r_{L B}^{(1,2)}, x_{L B}^{(1,2)}$ and $g_{(p)}$ are given by (46), (47), (48) and (25).

Now, let's find the corresponding lengths for the second stub:

c) Short circuit (stub)

$$
x_{S 2}^{(1,2,3,4)}=k_{2} n_{\left(\frac{S}{C}\right) S 2}^{(1,2,3,4)}
$$

or

$$
n_{\left(\frac{S}{C}\right) s 2}^{(1,2,3,4)}=\frac{x_{S 2}^{(1,2,3,4)}}{k_{2}}
$$




$$
l_{\left(\frac{S}{C}\right) S 2}^{(1,2,3,4)}=\frac{\lambda}{2 \pi} \operatorname{atan}\left[n_{\left(\frac{S}{C}\right) s 2}^{(1,2,3,4)}\right]
$$

d) Open circuit (stub)

$$
x_{S 2}^{(1,2,3,4)}=-\frac{k_{2}}{n_{\left(\frac{O}{C}\right) S 2}^{(1,2,3,4)}}
$$

or

$$
-\frac{k_{2}}{x_{S 2}^{(1,2,3,4)}}=n_{\left(\frac{O}{C}\right) S 2}^{(1,2,3,4)}
$$

$$
l_{\left(\frac{O}{C}\right) s 2}^{(1,2,3,4)}=\frac{\lambda}{2 \pi} \operatorname{atan}\left[n_{\left(\frac{O}{C}\right) s 2}^{(1,2,3,4)}\right]
$$

Finally, the reactance of the third stub can be obtained from (19),

$$
x_{S 3}^{(1,2,3,4)}==-\frac{p\left(1-r_{L B}^{(1,2) 2}-X_{2}^{(1,2,3,4) 2}\right)+X_{2}^{(1,2,3,4)}\left(1-p^{2}\right)}{\left(1-p X_{2}^{(1,2,3,4)}\right)^{2}+p^{2} r_{L B}^{(1,2) 2}}
$$

where

$r_{L B}^{(1,2)}$ is given by (46).

The corresponding lengths for the third stub are:

e) Short circuit (stub)

$$
x_{S 3}^{(1,2,3,4)}=k_{3} q_{\left(\frac{S}{C}\right) S 3}^{(1,2,3,4)}
$$

or

$$
q_{\left(\frac{S}{C}\right) S 3}^{(1,2,3,4)}=\frac{x_{S 3}^{(1,2,3,4)}}{k_{3}}
$$

$$
l_{\left(\frac{S}{C}\right) S 3}^{(1,2,3,4)}=\frac{\lambda}{2 \pi} \operatorname{atan}\left[q_{\left(\frac{S}{C}\right) S 3}^{(1,2,3,4)}\right]
$$

f) Open circuit (stub)

$$
x_{S 3}^{(1,2,3,4)}=-\frac{k_{3}}{q_{\left(\frac{O}{C}\right) S 3}^{(1,2,3,4)}}
$$

or

$$
-\frac{x_{S 3}^{(1,2,3,4)}}{k_{3}}=q_{\left(\frac{O}{C}\right) S 3}^{(1,2,3,4)}
$$

$$
l_{\left(\frac{O}{C}\right) s 2}^{(1,2,3,4)}=\frac{\lambda}{2 \pi} \operatorname{atan}\left[q_{\left(\frac{O}{C}\right) s 3}^{(1,2,3,4)}\right]
$$


For stub lengths the following formula must be respected,

$$
l_{s}=\left\{\begin{array}{lc}
\frac{\lambda}{2 \pi} \operatorname{atn}[P]+\frac{\lambda}{2} & \text { for } P<0 \\
\frac{\lambda}{2 \pi} \operatorname{atn}[P] & \text { for } P>0
\end{array}\right\}
$$

In the Table 1. the corresponding solutions are summarized as follows,

Table 1 The stub lengths in the triple stub matching.

\begin{tabular}{|c|c|c|}
\hline$l_{S 1}$ & $l_{S 2}$ & $l_{S 3}$ \\
\hline$l_{S 1}^{(1)}$ & $l_{S 2}^{(1)}$ & $l_{S 3}^{(1)}$ \\
\hline$l_{S 1}^{(2)}$ & $l_{S 2}^{(2)}$ & $l_{S 3}^{(2)}$ \\
\hline$l_{S 1}^{(3)}$ & $l_{S 2}^{(3)}$ & $l_{S 3}^{(3)}$ \\
\hline$l_{S 1}^{(4)}$ & $l_{S 2}^{(4)}$ & $l_{S 3}^{(4)}$ \\
\hline
\end{tabular}

This schema is valuable either for the short or the open stubs. Thus, the eight possible combinations can be given by this schema ((S/S/S; S/S/O; O/S/S; O/S/O/; S/O/S; S/O/0; 0/0/S; 0/0/0).

It is recommended to choose the shortest stub lengths because the shorter lengths permit a significantly better bandwidth.

$$
\begin{gathered}
l_{s 1}=\min \left(l_{s 1}^{(1)}, l_{s 1}^{(2)}, l_{s 1}^{(3)}, l_{s 1}^{(4)}\right) \\
l_{s 2}=\min \left(l_{s 2}^{(1)}, l_{s 2}^{(2)}, l_{s 2}^{(3)}, l_{s 2}^{(4)}\right) \\
l_{s 3}=\min \left(l_{s 3}^{(1)}, l_{s 3}^{(2)}, l_{s 3}^{(3)}, l_{s 3}^{(4)}\right)
\end{gathered}
$$

In the papers which treat the triple stub matching many authors after finding the lengths of the first stub use it for solving the rest of the problem as the double stub matching. In this paper as

it is proposed after finding the length of the first stub, the lengths of the second and the third stubs are simple to find directly from the corresponding formulas. Moreover, in this paper the characteristic impedance of each circuit is different.

- $\quad$ Special cases

a1) $d_{1}=d_{2}=d_{3}=\lambda / 4$

$$
\boldsymbol{a}=\boldsymbol{m}=\boldsymbol{p}=\infty
$$

$$
\begin{aligned}
& r_{L A}=\frac{p_{L}}{p_{L}^{2}+q_{L}^{2}}, \quad x_{L A}=-\frac{q_{L}}{p_{L}^{2}+q_{L}^{2}} \\
& r_{L B 1}=r_{L B 2}=\frac{r_{L A}}{X_{1(1)}^{2}+r_{L A}^{2}}=r_{L B} \leq 1 \\
& x_{L B 1}=-\frac{X_{1(1)}}{X_{1(1)}^{2}+r_{L A}^{2}}, \quad x_{L B 2}=-\frac{X_{1(2)}}{X_{1(2)}^{2}+r_{L A}^{2}}
\end{aligned}
$$




$$
\begin{aligned}
& X_{1}^{(1,2)}=r_{L A}+x_{S 1}^{(1,2)} \\
& x_{S 1}^{(1)}=-x_{L A}+r_{L A} \sqrt{Q t-1} \\
& x_{S 1}^{(2)}=-x_{L A}-r_{L A} \sqrt{Q t-1} \\
& Q=\frac{1}{r_{L A}} \\
& x_{S 2}^{(1,2,3,4)}=-x_{L B}^{(1,2)} \pm r_{L B} \sqrt{\frac{1}{r_{L B}}-1} \\
& x_{S 3}^{(1,2,3,4)}=-\frac{X_{2}^{(1,2,3,4)}}{\left(B_{2}^{(1,2,3,4)}\right)^{2}+r_{L B}^{(1,2) 2}}
\end{aligned}
$$

a2) $d_{1}=d_{2}=\lambda / 4, d_{3} \neq \lambda / 4$

We use (59)-(64)

$$
\boldsymbol{a}=\boldsymbol{m}=\infty, \quad \boldsymbol{p} \neq \infty
$$

$$
Q=\frac{r(p)}{r_{L A}}
$$

and (49) and (52) for $x_{S 2}^{(1,2,3,4)}$ and $x_{S 3}^{(1,2,3,4)}$

a3) $d_{1}=d_{3}=\lambda / 4, d_{2} \neq \lambda / 4$

$$
\boldsymbol{a}=\boldsymbol{p}=\infty, \quad \boldsymbol{m} \neq \infty
$$

We use (56) for $r_{L A}, x_{L A},(46)$, (48) and (58) for $r_{L B 1}, r_{L B 2}, x_{L B 1}, x_{L B 2}$, (3) (40) and (41) for $X_{1}^{(1,2)}, X_{S 1}^{(1,2)}$. For $x_{S 2}^{(1,2,3,4)}$ and $x_{S 3}^{(1,2,3,4)}(63)$ and (64) are used respectively with,

$$
Q=\frac{r(m)}{r_{L A}}
$$

a4) $d_{1}=\frac{\lambda}{4}, d_{2} \neq \frac{\lambda}{4} d_{3} \neq \frac{\lambda}{4}$

$$
\boldsymbol{a}=\infty, \quad \boldsymbol{m} \neq \infty, \quad \boldsymbol{p} \neq \infty
$$

We use (59) for $r_{L A}, x_{L A}$. All other expressions for $x_{S 1}^{(1,2)}, x_{S 2}^{(1,2,3,4)}$ and $x_{S 3}^{(1,2,3,4)}$ are calculated by the same expressions as in the general case.

a5) $d_{1} \neq \frac{\lambda}{4}, d_{2}=\lambda / 4 \quad d_{3} \neq \lambda / 4$

$$
\boldsymbol{a} \neq \infty, \quad \boldsymbol{m}=\infty, \quad \boldsymbol{p} \neq \infty
$$

We use (60) and (61) for calculating $x_{S 1}^{(1,2)},(57)$ and (58) for $r_{L B}, x_{L B 1}, x$ with 


$$
Q=\frac{1}{r(p) r_{L B}}
$$

All other expressions for $x_{S 2}^{(1,2,3,4)}$ and $x_{S 3}^{(1,2,3,4)}$ are calculated by the same expressions as in the general case.

a6) $d_{1} \neq \frac{\lambda}{4}, d_{2} \neq \frac{\lambda}{4} d_{3}=\lambda / 4$

$$
\boldsymbol{a} \neq \infty, \quad \boldsymbol{m} \neq \infty, \quad \boldsymbol{p}=\infty
$$

We use (63) and (64) for calculating $x_{S 2}^{(1,2,3,4)}$ and $x_{S 3}^{(1,2,3,4)}$ with (66). All other expressions are calculated by the same expressions as in the general case.

The stub lengths either for short circuit or open circuit are calculated by the previous expressions.

\section{Numerical validation}

In the literature is difficult to find the representative examples for triple stub matching where the stabs are in series with the transmission line. We give some examples which results will be verified by the coefficient SWR (standing wave ratio). These examples could be interesting as the benchmark problems.

\subsection{Example}

The terminating impendence is $Z_{L}=(50-j 10) \Omega$ and the characteristic impendence $Z_{0}$ of the line and the stubs is 50 $\Omega$. The first stub is connected to the load $\left(\mathrm{d}_{1}=0 \lambda\right)$. The spacing between the first and second stub is stub $\mathrm{d}_{2}=1 / 8 \lambda$ and between the third and the second stub is $d_{3}=1 / 8 \lambda$. Determine the lengths of the short-circuited stubs when the match is achieved.

Solution:

$$
r_{L A}=1, \quad x_{L A}=-0.2, \quad Q=1, \quad t_{\max }=1
$$

There is not the unique solution.

We begin by $t=1$ for which,

Table 2 The stub lengths in the triple stub matching (S/C), $t=1$

\begin{tabular}{|c|c|c|}
\hline $\boldsymbol{l}_{\boldsymbol{s} \mathbf{1}}$ & $\boldsymbol{l}_{\boldsymbol{s} \mathbf{2}}$ & $\boldsymbol{l}_{\boldsymbol{s} \mathbf{3}}$ \\
\hline 0.1394 & 0.1762 & 0.1250 \\
\hline 0.1394 & 0.1762 & 0.1250 \\
\hline 0.1394 & 0.1762 & 0.1250 \\
\hline 0.1394 & 0.1762 & 0.1250 \\
\hline
\end{tabular}

Table 3 The stub lengths in the triple stub matching $(\mathrm{S} / \mathrm{C}), \mathrm{t}=3$.

\begin{tabular}{|c|c|c|}
\hline $\boldsymbol{l}_{\boldsymbol{S} \mathbf{1}}$ & $\boldsymbol{l}_{\boldsymbol{S} \mathbf{2}}$ & $\boldsymbol{l}_{\boldsymbol{S} \mathbf{3}}$ \\
\hline 0.1919 & 0.2099 & 0.1875 \\
\hline 0.1919 & 0.1762 & 0.4375 \\
\hline 0.4664 & 0.1762 & 0.1875 \\
\hline 0.4664 & 0.0181 & 0.4375 \\
\hline
\end{tabular}


Table 4 The stub lengths in the triple stub matching (S/C), $t=10$.

\begin{tabular}{|c|c|c|}
\hline $\boldsymbol{l}_{\boldsymbol{S 1}}$ & $\boldsymbol{l}_{\boldsymbol{S 2}}$ & $\boldsymbol{l}_{\boldsymbol{S 3}}$ \\
\hline 0.2128 & 0.2018 & 0.2110 \\
\hline 0.2128 & 0.1762 & 0.3238 \\
\hline 0.3307 & 0.1762 & 0.2110 \\
\hline 0.3307 & 0.1074 & 0.3238 \\
\hline
\end{tabular}

Table 5 The stub lengths in the triple stub matching (S/C), $t=100$.

\begin{tabular}{|c|c|c|}
\hline $\boldsymbol{l}_{\boldsymbol{S 1}}$ & $\boldsymbol{l}_{\boldsymbol{S 2}}$ & $\boldsymbol{l}_{\boldsymbol{S} \mathbf{3}}$ \\
\hline 0.2358 & 0.1871 & 0.2355 \\
\hline 0.2358 & 0.1762 & 0.2677 \\
\hline 0.2681 & 0.1762 & 0.2355 \\
\hline 0.2681 & 0.1612 & 0.2677 \\
\hline
\end{tabular}

Table 6 The stub lengths in the triple stub matching (S/C), $t=100000$.

\begin{tabular}{|c|c|c|}
\hline $\boldsymbol{l}_{\boldsymbol{S} \mathbf{1}}$ & $\boldsymbol{l}_{\boldsymbol{S} \mathbf{2}}$ & $\boldsymbol{l}_{\boldsymbol{S} \mathbf{3}}$ \\
\hline 0.2495 & 0.1766 & 0.2495 \\
\hline 0.2495 & 0.1762 & 0.2505 \\
\hline 0.2505 & 0.1762 & 0.2495 \\
\hline 0.2505 & 0.1758 & 0.2505 \\
\hline
\end{tabular}

Table 7 The stub lengths in the triple stub matching (S/C), $t=10^{10}$

\begin{tabular}{|c|c|c|}
\hline $\boldsymbol{l}_{\boldsymbol{S 1}}$ & $\boldsymbol{l}_{\boldsymbol{S} \mathbf{2}}$ & $\boldsymbol{l}_{\boldsymbol{S} \mathbf{3}}$ \\
\hline 0.2500 & 0.1762 & 0.2500 \\
\hline 0.2500 & 0.1762 & 0.2500 \\
\hline 0.2500 & 0.1762 & 0.2500 \\
\hline 0.2500 & 0.1762 & 0.2500 \\
\hline
\end{tabular}

From Tables 2 to 7 we can see that for different ' $t$ ' there is not the unique solution for the stub matching, but each solution satisfies the matching condition (34) that will be proved by the coefficient SWR (APPENDIX). We choose randomly the lengths of stub1,2 and 3.

Not unique solution: $t=10$ (Second row in Table 4)

I) SWR before the load:

$$
\begin{gathered}
d_{1 z N}=0, \quad l_{1 z N}=0 \\
z_{1 N}=(50-j 10) \Omega \\
\left|\Gamma_{\text {BLoad }}\right|=0.0995, \quad S W R_{\text {BLoad }}=1.2210
\end{gathered}
$$

II) SWR between the first and the second stub: 


$$
\begin{gathered}
d_{1}=0, \quad l_{S 1}^{(1)}=0.2128 \lambda \\
z_{\text {Totals } 1}=(50+j 200.01) \Omega \\
\left|\Gamma_{(S 1-S 2)}\right|=0.8944, \quad S W R_{(S 1-S 2)}=17.9456
\end{gathered}
$$

III) VSWR after the second stub:

$$
\begin{gathered}
z_{\text {Total } S 2}=(9.9988+j 19.9887) \Omega \\
d_{2}=0.125 \lambda, \quad l_{S 2}^{(1)}=0.1762 \lambda \\
\left|\Gamma_{(S 2-S 3)}\right|=0.7071, \quad S W R_{(S 2-S 3)}=5.8282
\end{gathered}
$$

IV) VSWR after the third stub:

$$
\begin{gathered}
z_{T S 3}=(49.9613-j 0.0287) \Omega \\
d_{3}=0.125 \lambda, \quad l_{S 3}^{(1)}=0.3238 \lambda \\
\left|\Gamma_{(A S 3)}\right|=4.8200 \cdot 10^{-4} \\
S W R_{(A S 3)}=1.0010
\end{gathered}
$$

Thus, for any ' $\mathrm{t}^{\prime}(34)$ is satisfied.

\subsection{Examples}

The terminating impendence is $Z_{L}=(300+j 100) \Omega$ and the characteristic impendence $Z_{0}$ of the line and the stubs is 50 $\Omega$. The first stub is away $\mathrm{d}_{1}=0.503 \lambda$ from the load. The spacing between the first and second stub is stub $\mathrm{d}_{2}=3 / 8 \lambda$ and between the third and the second stub is $d_{3}=3 / 8 \lambda$. Determine the lengths of the short-circuited stubs when the match is achieved.

Solution:

$$
\begin{gathered}
r_{L A}=6.3934, \quad x_{L A}=1.3465, \quad Q=0.1564 \\
t_{\max }=6.3934
\end{gathered}
$$

For any ' $t$ ' between 1 and 6.3934 we have the unique solution for the stub matching (Table 8).

Short stubs:

Table 8 The stub lengths (S/C), $\mathbf{1} \leq \boldsymbol{t} \leq \mathbf{6 . 3 9 3 4}$.

\begin{tabular}{|c|c|c|}
\hline $\boldsymbol{l}_{\boldsymbol{S} \mathbf{1}}$ & $\boldsymbol{l}_{\boldsymbol{S} \mathbf{}}$ & $\boldsymbol{l}_{\boldsymbol{S} \mathbf{}}$ \\
\hline 0.3141 & 0.3559 & 0.1470 \\
\hline 0.3141 & 0.3059 & 0.2965 \\
\hline 0.3141 & 0.3559 & 0.1470 \\
\hline 0.3141 & 0.3059 & 0.2965 \\
\hline
\end{tabular}


Table 9 The stub lengths $(\mathrm{S} / \mathrm{C}), \mathrm{t}=6.4$

\begin{tabular}{|c|c|c|}
\hline $\boldsymbol{l}_{\boldsymbol{S} \mathbf{1}}$ & $\boldsymbol{l}_{\boldsymbol{S 2}}$ & $\boldsymbol{l}_{\boldsymbol{S 3}}$ \\
\hline 0.3196 & 0.3565 & 0.1470 \\
\hline 0.3196 & 0.3061 & 0.2965 \\
\hline 0.3094 & 0.3553 & 0.1470 \\
\hline 0.3094 & 0.3058 & 0.2965 \\
\hline
\end{tabular}

Table 10 The stub lengths (S/C), $t=100$.

\begin{tabular}{|c|c|c|}
\hline $\boldsymbol{l}_{\boldsymbol{S} \mathbf{1}}$ & $\boldsymbol{l}_{\boldsymbol{S} \boldsymbol{2}}$ & $\boldsymbol{l}_{\boldsymbol{S} \mathbf{3}}$ \\
\hline 0.2428 & 0.3336 & 0.2323 \\
\hline 0.2428 & 0.3201 & 0.2645 \\
\hline 0.2559 & 0.3279 & 0.2323 \\
\hline 0.2559 & 0.3159 & 0.2645 \\
\hline
\end{tabular}

Table 11 The stub lengths (S/C), $\mathrm{t}=10^{10}$

\begin{tabular}{|c|c|c|}
\hline $\boldsymbol{l}_{\boldsymbol{s} \mathbf{1}}$ & $\boldsymbol{l}_{\boldsymbol{S 2}}$ & $\boldsymbol{l}_{\boldsymbol{s} \mathbf{3}}$ \\
\hline 0.2500 & 0.3238 & 0.2500 \\
\hline 0.2500 & 0.3238 & 0.2500 \\
\hline 0.2500 & 0.3238 & 0.2500 \\
\hline 0.2500 & 0.3238 & 0.2500 \\
\hline
\end{tabular}

We have two types of matching solutions (see Tables 8 to 11):

a) Unique for $1 \leq t \leq t_{\max }=6.3934$

b) Not unique for $t>t_{\max }=6.3934$

Unique solution: $1 \leq t \leq t_{\max }=6.3934$ (First row in Table 8)

I) SWR before the load:

$$
\begin{gathered}
d_{1 z N}=0, \quad l_{1 z N}=0 \\
z_{1 N}=(300+j 100) \Omega \\
\left|\Gamma_{\text {BLoad }}\right|=0.7397, \quad S W R_{\text {BLoad }}=6.6837
\end{gathered}
$$

II) SWR between the first and the second stub:

$$
\begin{gathered}
d_{1}=0.503 \lambda, \quad l_{S 1}^{(1)}=0.3141 \lambda \\
z_{\text {Totals } 1}=(319.67-j 50.036) \Omega \\
\left|\Gamma_{(S 1-S 2)}\right|=0.7352, \quad S W R_{(S 1-S 2)}=6.5538
\end{gathered}
$$

III) VSWR after the second stub:

$$
z_{\text {Totals } 2}=(15.6411-j 13.7106) \Omega
$$




$$
\begin{gathered}
d_{2}=0.375 \lambda, \quad l_{S 2}^{(1)}=0.3559 \lambda \\
\left|\Gamma_{(S 2-S 3)}\right|=0.5517, \quad S W R_{(S 2-S 3)}=3.4610
\end{gathered}
$$

IV) VSWR after the third stub:

$$
\begin{gathered}
z_{T S 3}=(50.0815-j 0.0358) \Omega \\
d_{3}=0.375 \lambda, \quad l_{S 3}^{(1)}=0.1470 \lambda \\
\left|\Gamma_{(A S 3)}\right|=8.8948 \cdot 10^{-4} \\
S W R_{(A S 3)}=1.0018
\end{gathered}
$$

No unique solution: $t=100>t_{\max }=6.3934$ (Fourth row in Table 10 )

I) SWR before the load:

$$
\begin{gathered}
d_{1 z N}=0, \quad l_{1 z N}=0 \\
z_{1 N}=(300+j 100) \Omega \\
\left|\Gamma_{\text {BLoad }}\right|=0.7397, \quad V S W R_{\text {BLoad }}=6.6837
\end{gathered}
$$

II) SWR between the first and the second stub:

$$
\begin{gathered}
d_{1}=0.503 \lambda, \quad l_{S 1}^{(1)}=0.2559 \lambda \\
z_{\text {Totals } 1}=(319.67-j 1280.8) \Omega \\
\left|\Gamma_{(S 1-S 2)}\right|=0.9818, \quad V S W R_{(S 1-S 2)}=109.1800
\end{gathered}
$$

III) VSWR after the second stub:

$$
\begin{gathered}
z_{\text {Totals2 }}=(0.9884-j 59.9680) \Omega \\
d_{2}=0.375 \lambda, \quad l_{S 2}^{(1)}=0.3159 \lambda \\
\left|\Gamma_{(S 2-S 3)}\right|=0.9839, \quad V S W R_{(S 2-S 3)}=123.3619
\end{gathered}
$$

IV) VSWR after the third stub:

$$
\begin{gathered}
z_{T S 3}=(49.2535-j 0.5696) \Omega \\
d_{3}=0.375 \lambda, \quad l_{S 3}^{(1)}=0.2645 \lambda \\
\left|\Gamma_{(A S 3)}\right|=0.0095 \\
\operatorname{VSWR}_{(A S 3)}=1.0191
\end{gathered}
$$

Thus, for any ' $\mathrm{t}^{\prime}(34)$ is satisfied.

\subsection{Example}

A load with a load impedance of $Z_{L}=(250+j 80) \Omega$ is to be matched to a transmission line of a microstrip line with a characteristic input impedance of $50 \Omega$ using a triple stub. All stubs are open with the same characteristic impedances 
as the transmission line. The gaps between the stubs are $d_{2}=1 / 8 \lambda$ and $d_{3}=3 / 8 \lambda$, and the gap between the load and the first stub is fixed at $d_{1}=0.482 \lambda$.

Solution:

In this case,

$$
\begin{aligned}
r_{L A}=2.9462, \quad x_{L A} & =2.9462, \quad Q=0.3394 \\
t_{\max } & =2.9462
\end{aligned}
$$

This is the case of the unique solution where all expressions do not depend on the parameter ' $t$ ', (37) and (38) for,

$$
1 \leq t \leq t_{\max }=2.9462
$$

For any ' $t$ ' from the interval $1 \leq t \leq t_{\max }=2.9462$ the unique solution is given for the open and the short stubs in Table 12.

Table 12 The stub lengths (O/C).

\begin{tabular}{|c|c|c|}
\hline $\boldsymbol{l}_{\boldsymbol{S 1}}$ & $\boldsymbol{l}_{\boldsymbol{S} \mathbf{2}}$ & $\boldsymbol{l}_{\boldsymbol{S} \mathbf{3}}$ \\
\hline 0.0857 & 0.3707 & 0.3099 \\
\hline 0.0857 & 0.1293 & 0.0629 \\
\hline 0.0857 & 0.3707 & 0.3099 \\
\hline 0.0857 & 0.1293 & 0.0629 \\
\hline
\end{tabular}

For any ' $t$ ' from the interval $t=t_{\max }>2.9462$ we obtain the different solutions as it was shown in the previous examples. The matching condition (34) is satisfied.

\subsection{Example}

Let's solve the following problem where the terminating impendence is $Z_{L}=(25-j 25) \Omega$ and the characteristic impendence $Z_{0}$ of the line and the stubs is $50 \Omega$. The first stub is away $\mathrm{d}_{1}=0.25 \lambda$ from the load. The spacing between the first and second stub is stub $\mathrm{d}_{2}=1 / 4 \lambda$ and between the third and the second stub is $\mathrm{d}_{3}=1 / 4 \lambda$. Determine the lengths of the open circuited stubs when the match is achieved.

Solution:

This is the special case a1)

$$
\begin{gathered}
r_{L A}=0.0917, \quad x_{L A}=-0.0200, \quad Q=10.9039 \\
t_{\max }=0.0917<1
\end{gathered}
$$

There is not the unique solution but for any ' $t$ ' the matching condition (34) is satisfied.

For $t=3$ (Table 13)

Table 13 The stub lengths (O/C)

\begin{tabular}{|c|c|c|}
\hline $\boldsymbol{l}_{\boldsymbol{S 1}}$ & $\boldsymbol{l}_{\boldsymbol{S} \mathbf{2}}$ & $\boldsymbol{l}_{\boldsymbol{S 3}}$ \\
\hline 0.3125 & 0.3703 & 0.4020 \\
\hline 0.3125 & 0.2500 & 0.0980 \\
\hline 0.0625 & 0.2500 & 0.4020 \\
\hline 0.0625 & 0.1297 & 0.0980 \\
\hline
\end{tabular}




\subsection{Examples}

Finally let's solve the following example in which all characteristic impedances are different.

The terminating impendence is $Z_{L}=(60-j 80) \Omega$ and the characteristic impendence of the line $Z_{0}$ is $50 \Omega$. The characteristic impedances of stubs are respectively $Z_{S 1}=75 \Omega, Z_{S 2}=100 \Omega$ and $Z_{S 3}=125 \Omega$. The first stub is away $\mathrm{d}_{1}=$ $0.154 \lambda$ from the load. The spacing between the first and second stub is stub $\mathrm{d}_{2}=3 / 8 \lambda$ and between the third and the second stub is $d_{3}=1 / 8 \lambda$. Determine the lengths of the open stubs as well as the short-circuited stubs when the match is achieved.

Solution:

We start with the open stubs and $x=5$ for which we have,

$$
\begin{gathered}
r_{L A}=0.2649, x_{L A}=-0.1835, Q=3.7746 \\
t_{\max }=0.2649<1
\end{gathered}
$$

Thus, there is not the unique solution. The results for $x=5$ are given in Table 14 .

Table 14 The stub lengths $(\mathrm{O} / \mathrm{C})$

\begin{tabular}{|c|c|c|}
\hline $\boldsymbol{l}_{\boldsymbol{S} \mathbf{1}}$ & $\boldsymbol{l}_{\boldsymbol{S} \mathbf{}}$ & $\boldsymbol{l}_{\boldsymbol{S} \mathbf{3}}$ \\
\hline 0.2818 & 0.3923 & 0.3894 \\
\hline 0.2818 & 0.3167 & 0.1894 \\
\hline 0.1049 & 0.1833 & 0.3894 \\
\hline 0.1049 & 0.1077 & 0.1894 \\
\hline
\end{tabular}

Let us verify these results by the coefficient SWR for the shortest lengths of stubs (Fourth row in Table 14).

I) SWR before the load:

$$
\begin{gathered}
d_{1 z N}=0, \quad l_{1 z N}=0 \\
z_{1 N}=(60-j 80) \Omega \\
\left|\Gamma_{\text {BLoad }}\right|=0.5927, S W R_{\text {BLoad }}=3.9110
\end{gathered}
$$

II) SWR between the first and the second stub:

$$
\begin{gathered}
d_{1}=0.154 \lambda, \quad l_{S 1}^{(1)}=0.1049 \lambda \\
z_{\text {Total } 1}=(10.8822-j 9.7414) \Omega \\
\left|\Gamma_{(S 1-S 2)}\right|=0.9089, \quad S W R_{(S 1-S 2)}=20.9535
\end{gathered}
$$

III) VSWR after the second stub:

$$
\begin{gathered}
z_{\text {Totals } 2}=(20.0070+j 10.0690) \Omega \\
d_{2}=0.375 \lambda, \quad l_{S 2}^{(1)}=0.1077 \lambda \\
\left|\Gamma_{(S 2-S 3)}\right|=0.4473, \quad S W R_{(S 2-S 3)}=2.6187
\end{gathered}
$$

IV) VSWR after the third stub

$$
z_{T S 3}=(50.1488+j 0.0526 \mathrm{i}) \Omega
$$




$$
\begin{gathered}
d_{3}=0.125 \lambda, \quad l_{S 3}^{(1)}=0.1894 \lambda \\
\left|\Gamma_{(A S 3)}\right|=0.0016 \\
S W R_{(A S 3)}=1.0032
\end{gathered}
$$

Short stubs:

Now, for the short stubs and $x=5$ the results are given in Table 15 .

Table 15 The stub lengths (S/C)

\begin{tabular}{|c|c|c|}
\hline $\boldsymbol{l}_{\boldsymbol{S} \mathbf{1}}$ & $\boldsymbol{l}_{\boldsymbol{S} \mathbf{2}}$ & $\boldsymbol{l}_{\boldsymbol{S} \mathbf{3}}$ \\
\hline 0.0318 & 0.1423 & 0.1394 \\
\hline 0.0318 & 0.0667 & 0.4394 \\
\hline 0.3549 & 0.4333 & 0.1394 \\
\hline 0.3549 & 0.3577 & 0.4394 \\
\hline
\end{tabular}

$$
\begin{gathered}
r_{L A}=0.2649, x_{L A}=-0.1835, Q=3.7746 \\
t_{\max }=0.2649
\end{gathered}
$$

Let us verify these results by the coefficient SWR (Second row in Table 15).

I) SWR before the load:

$$
\begin{gathered}
d_{1 z N}=0, \quad l_{1 z N}=0 \\
Z_{1}=(60-j 80) \Omega \\
\left|\Gamma_{\text {BLoad }}\right|=0.5927, S W R_{\text {BLoad }}=3.9110
\end{gathered}
$$

II) SWR between the first and the second stub:

$$
\begin{gathered}
d_{1}=0.154 \lambda, \quad l_{S 1}^{(1)}=0.0318 \lambda \\
z_{\text {Totals } 1}=(13.2464+j 6.0124) \Omega \\
\left|\Gamma_{(S 1-S 2)}\right|=0.5862, \quad S W R_{(S 1-S 2)}=3.8333
\end{gathered}
$$

III) VSWR after the second stub:

$$
\begin{gathered}
z_{\text {Totals } 2}=(19.9924+j 10.0100) \Omega \\
d_{2}=0.375 \lambda, \quad l_{S 2}^{(1)}=0.0667 \lambda \\
\left|\Gamma_{(S 2-S 3)}\right|=0.4474, \quad S W R_{(S 2-S 3)}=2.6192
\end{gathered}
$$

IV) VSWR after the third stub:

$$
\begin{gathered}
z_{T S 3}=(50.0086-j 0.0067) \Omega \\
d_{3}=0.125 \lambda, \quad l_{S 3}^{(1)}=0.4394 \lambda
\end{gathered}
$$




$$
\begin{gathered}
\left|\Gamma_{(A S 3)}\right|=1.0914 \cdot 10^{-4} \\
S W R_{(A S 3)}=1.0002
\end{gathered}
$$

Thus, in all examples is confirmed by SWR the validity of obtained results. In all previous example the software [11] is used.

\section{Appendix}

\subsection{Determination of the standing wave ratio SWR}

$$
\begin{gathered}
a=\tan (\beta d), \quad b=\tan (\beta l) \\
Z_{(d)}=Z_{0} \frac{Z_{0}+j Z_{0} \tan \left(\beta d_{1}\right)}{Z_{0}+j \tan \left(\beta d_{1}\right) Z_{0}}=Z_{0} \frac{Z_{0}+j Z_{0} a}{Z_{0}+j \tan Z_{L} a}
\end{gathered}
$$

Short stub:

$$
Z_{(S-l)}=j Z_{0} \tan (\beta l)=j Z_{0} b
$$

Open stub:

$$
Z_{(o-l)}=-j Z_{0} \operatorname{ctan}(\beta l)=-j Z_{0} / b
$$

Total impedance at the connexion,

$$
Z_{T C O N}=Z_{(d)}+Z_{(S-l)} \text { or } Z_{T C O N}=Z_{(d)}+Z_{(O-l)}
$$

The module of the coefficient of the reflexion $|\Gamma|$ at the connexion,

$$
|\Gamma|=\frac{\left|Z_{\text {TCON }}-Z_{0}\right|}{\left|Z_{\text {TCON }}+Z_{0}\right|}
$$

The standing wave ratio,

$$
S W R=\frac{1+|\Gamma|}{1-|\Gamma|}
$$

\section{Conclusion}

In this paper we present the improved analytical approach for the triple stub matching. The stubs are in series with the transmission line and the load. All characteristic impedances either for the transmission line or for the stubs are different. The lengths of all stubs were found as well as the domains of the matching for the first and second stubs. Even though there is not the unique solution of the matching we show that under some matching conditions the unique solution is possible. The paper is written in detail with the general and the special cases. Many representative examples are solved in detail so that potential readers can easily follow and understand them. All results are verified by the standing wave ratio SWR which must be1 near the generator. It is recommended to verify these examples by using the Smith chart. In this paper we treated the short and open stubs bath other their combinations are possible. This paper is useful for the engineers and the physicians which work in this domain.

\section{Compliance with ethical standards}

\section{Disclosure of conflict of interest}

Authors declare no conflict of interest. 


\section{References}

[1] Orfanidis SJ. Electromagnetic Waves and Antennas, Rutgers University.

[2] Townsend. AAA. The Smith chart and its Applications, Ingeneria/TEM\%20//Material\%29Vario/Smith_Book_Complete.pdf.

[3] Pozar DM. 'Microwave Engineering', Fourth Edition.

[4] Jackson JD. Classical Electrodynamics, John Wiley and Sons Inc., Third Edition, New York. 1998.

[5] Benson FA, Benson TM. Fields, Waves and Transmission lines, Springer-Science \& Business Media, BV.

[6] Davidson SB. Computational Electromagnetics for RF and Microwave Engineering, 2nd Edition. November 2010.

[7] Collin RE. Foundations for Microwave Engineering, $2^{\text {nd }}$ Edition. January 2001.

[8] Kraus JD. Electromagnetics, Third edition McGraw-Hill Book Company.

[9] Collier R. Transmission lines, 1st Edition, ISBN-10: 1107026008.

[10] Balanis CA. 'Engineering Electromagnetics', ISBN: 0471621943.

[11] Babic S, Akyel C. Software- Matching (MATLAB). 2019. 